\title{
A Heart Valve Sample Collection for Sustainable Research in Indonesia
}

Ery Kus Dwianingsih ${ }^{1}$, Hasanah Mumpuni², Erika Maharani², Jajah Fachiroh ${ }^{3}$, Dyah Samti Mayasari²

${ }^{1}$ Department of Anatomical Pathology, ${ }^{2}$ Department of Cardiology, ${ }^{3}$ Department of Histology, Faculty of Medicine, Public Health and Nursing,

Universitas Gadjah Mada, Yogyakarta, Indonesia

\section{Statement of the Problem}

Rheumatic heart disease (RHD) is a permanent damage of the heart valves, commonly caused by rheumatic fever, preceded with infection of Streptococcus bacteria in sore throat or skin. This disease variously occurs in children and adult and still become major problem of valvular disease in developing country, specialy in Indonesia. ${ }^{1}$ Pathogenesis of RHD remains incompletely understood. Genetic predispotitions has been proposed as of important risk factor in RHD. Several genes have been associated with RHD through immune responses pathway, including TNF- $\alpha$, IL10 and IL4..$^{2,3}$ This heart valve collection is performed to elaborate genetic risk factor profile of RHD in Indonesian population for better therapy approach.

\section{Proposed Solution}

Heart valve of patients with RHD in Sardjito Hospital, Yogyakarta, are recruited after informed consent is completed. Formalin-Fixed Paraffin Embedded (FFPE) and fresh tissue of heart valve are collected for diagnosis and research purpose. Up to April 2018, 45 samples has been collected. FFPE samples are sliced for hematoxylin eosin staining and immunohistochemical (IHC) analysis to established the diagnosis. The left over sample will be stored to support sustainable research in elaborating genetic risk factor of RHD in Indonesia.

Target population of this heart valves collection is cardiology patients who are admitted for heart valve replacement due to heart valve impairment. The inclusion collection criteria are included: (1). Willingness of patients to participate in this heart valve collection (informed consent); (2). Availability of specimen collection in form of FFPE and fresh tissue samples. (3) Clinical variables, including demographic information (name, sex, and age), clinical data (hospital medical record number, pathological record number, and disease status), specimen information (date of specimens collection, type of specimens, date of processing). Exclusion criteria are included; (1) patients with congenital heart disease and (2) with infection other than rheumatic heart disease.

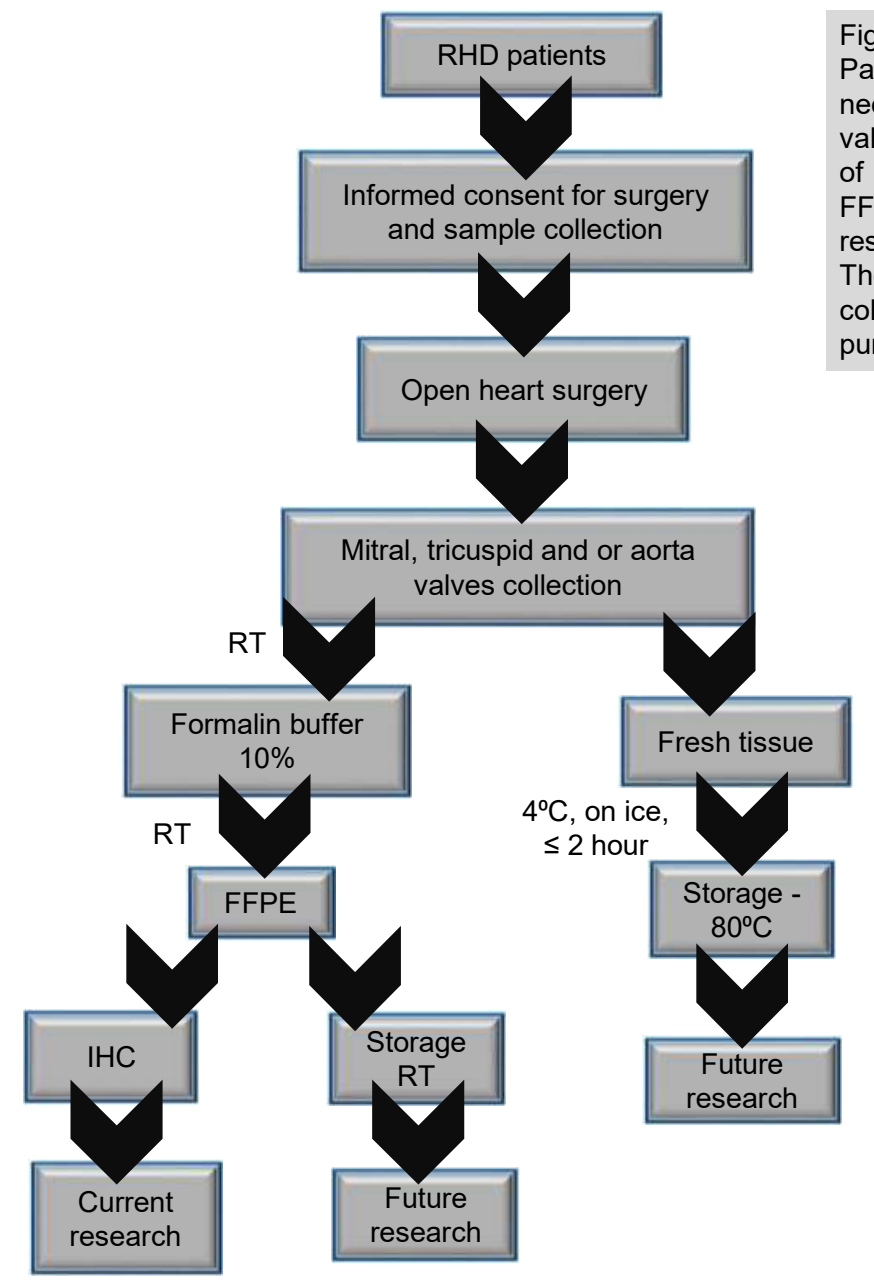

Figure 1. The flow of heart valves sample collection and storage. Patients clinically suspected with rheumatic heart disease and need open heart surgery is included into banking activity. Heart valves from mitral, tricuspid and or aorta are collected. Some part of sample is soaked with formalin buffer for FFPE block. The FFPE sampel is immunohistochemically stained for current research purpose to detect expression of TNF- $\alpha$, IL10 and IL4. The left over sample is stored in room temperature. Some part is collected in fresh manner to be kept in $-80^{\circ} \mathrm{C}$ for further research purpose. Room Temperature : RT

\section{Conclusion}

RHD remains major problem of valvular disease in developing country. The collection of heart valve will greatly contribute to unravel genetic risk factor of $\mathrm{RHD}$ and give benefit for future research in cardiology field in Indonesia.

\section{Reference}

1. Rodriguez-Fernandez R, Amiya R, Wyber $R$, et al (2015). Rheumatic heart disease among adults in a mining community of Papua, Indonesia: findings from an occupational cohort. Heart Asia, 7, 44-8.

2. Guilherme L, Cury P, Demarchi LM, et al (2004) Rheumatic heart disease: proinflammatory cytokines play a role in the progression and maintenance of valvular lesions. Am J Pathol, 165, 1583-91.

3. Guilherme L, Kohler KF, Kalil J (2011a). Rheumatic heart disease: mediation by complex immune events. Adv Clin Chem, 53, 31-50. 